\title{
Progress in application on the prediction of rolling bearing performances for urban rail and traffic engineering
}

\author{
Xin-Tao XIA ${ }^{1, a}$, Shu-Jing DONG ${ }^{1, b^{*}}$ and Yuan-Yuan QIN ${ }^{1, c}$ \\ ${ }^{1}$ Henan University of Science and Technology, Luoyang, P.R. China \\ axiaxt1957@163.com, b09101060206@163.com, ${ }^{\mathrm{c}} 136517162 @ q q . c o m$
}

\begin{abstract}
Keywords: Urban rail and traffic engineering. Rolling bearings. Poor information. Function polytrope Abstract. At present, an unprecedented development situation of the urban rail and traffic engineering is presented. Bearing as a key component of vehicle operation safety, its performances and reliability should achieve higher requirements. Because the number of characteristic data of bearing performances is small and prior information on probability distributions and trends is poor, statistics has difficulty in assessing the bearing performance. In order to study the nonlinear dynamic characteristics of rolling bearing performances, a unified representation of variable uncertainty and function polytrope characteristics of rolling bearing performances are proposed in information-poor relation space, which reveals its evolvement rule of nonlinear dynamic characteristics. The development of bearing technology for the urban rail and traffic Engineering should comply with safe operation, high speed, a longer maintenance period and the excellent running comfort.
\end{abstract}

\section{Introduction}

In recent years, as urban rail transit develops rapidly, the operation safety problem of railway highlights increasingly. The complexity of rail vehicles equipment is increasing, and the failure rate is rising, where the bearing failure is the major form of mechanical failures [1,2]. Therefore, how to predict the bearing performance of rail transit vehicles efficiently, rapidly and accurately is an important issue to be resolved.

The paper describes the method of analyzing bearing performance with poor information. Poor information means that characteristic information presented in the subject investigated is incomplete and insufficient, along with a small sample and the lack in prior knowledge. Experiments on rolling bearing performances concern primarily with statistical evaluation of the fatigue life $[3,4]$. By using fuzzy set theory, grey system theory, information entropy theory, and grey bootstrap theory [5,6 \& 7], some scholars have studied the performance of rolling bearings for the urban rail and transit. This paper presents a method of test evaluation for evolvement of nonlinear dynamic characteristics of rolling bearing performances using information-poor process theory to reveal, under the poor information condition, the new properties of nonlinearly dynamical evolvement of modern bearing performances.

Aiming at information-poor problems in performance test of rolling bearings, the variant gene is recognized, and the poor-information relation space is constructed; via theoretical and experimental studies, a unified representation of the variable uncertainty and function polytrope of bearing performances is provided in the information-poor space, so that assessment for evolvement of nonlinear dynamic characteristic of rolling bearing performances can be in progress $[8,9]$.

The paper offers a train of thought for breaking through the system of the statistical theory which is chronically adopted to evaluate rolling bearing performances for the urban rail and transit.

\section{Studies on variable uncertainty and function polytrope of rolling bearing performances}

The information entropy characteristics of rolling bearing performances. In 1948, C. E. Shannon proposed the concept of entropy, so that the problem of quantitative information can be solved. The concept of information entropy is used to describe the uncertainty of information sources, which can be defined as: 


$$
H(x)=-\sum_{i=1}^{n} p_{i}(x) \ln \left(p_{i}(x)\right)
$$

where, $i$ is the group number of variables $x, p_{i}(x)$ is the probability at the point of $x=x_{i}$, and $n$ is the number of groups in the data.

5 sets of large bearing are selected for experimental research, and bearing speed are respectively $3 \mathrm{r} / \mathrm{min}, 7 \mathrm{r} / \mathrm{min}$ and $11 \mathrm{r} / \mathrm{min}$. Data is accessed in the test bed of large bearing friction torque. Some experimental results are shown in Fig. (1, $2 \& 3)$, and the results of probability distribution of the friction torque are shown in Fig. $(4,5 \& 6)$.

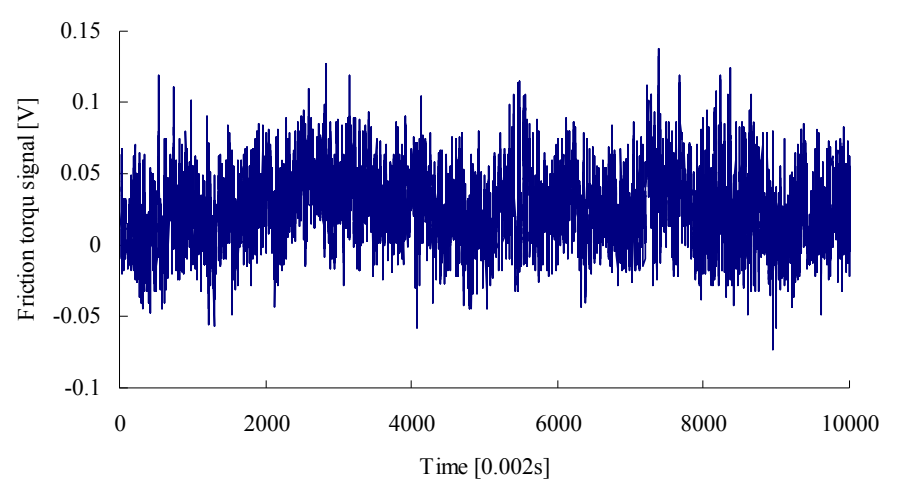

Fig. 1 Data sequence of friction torque (3r/min, Bearing1)

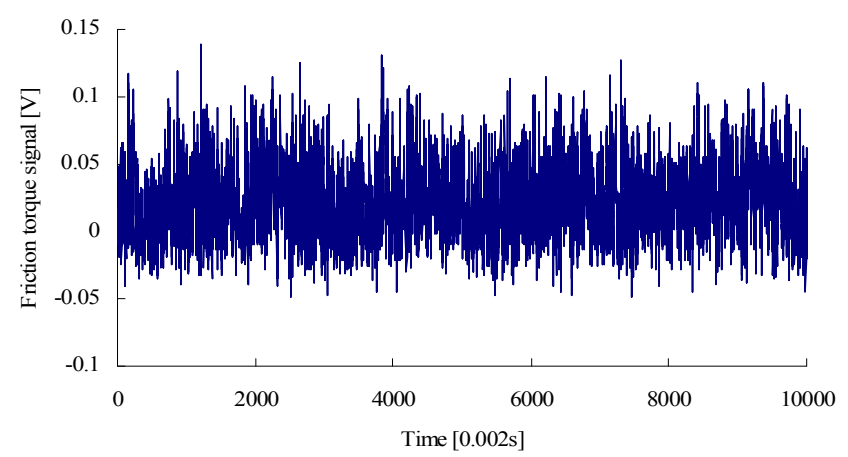

Fig. 2 Data sequence of friction torque ( $7 \mathrm{r} / \mathrm{min}$, Bearing1)

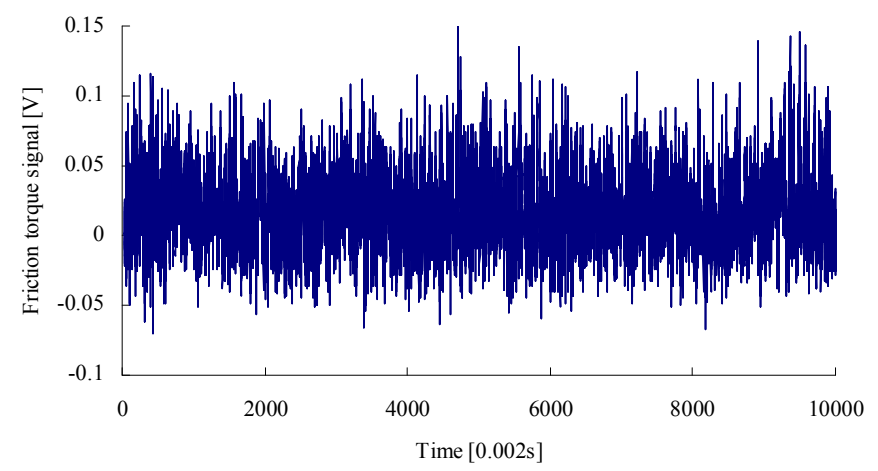

Fig. 3 Data sequence of friction torque (11r/min, Bearing1) 


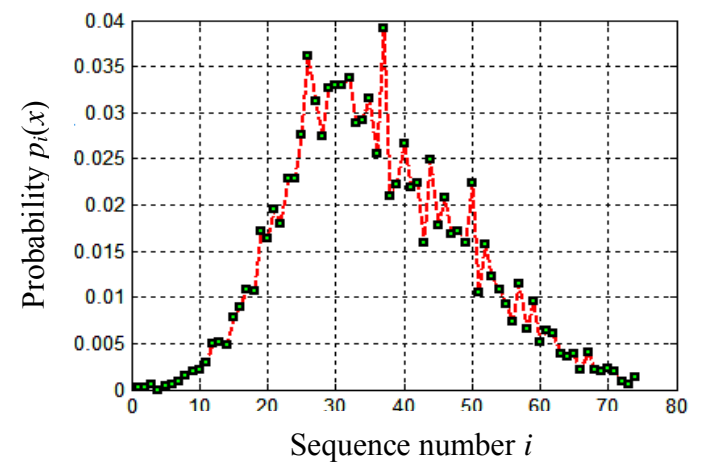

Fig. 4 Probability distribution of the friction torque (3r/min)

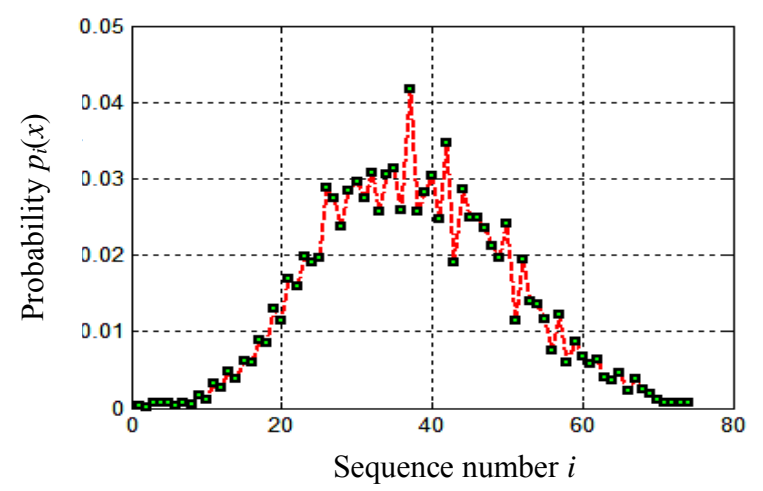

Fig. 5 Probability distribution of the friction torque $(7 \mathrm{r} / \mathrm{min})$

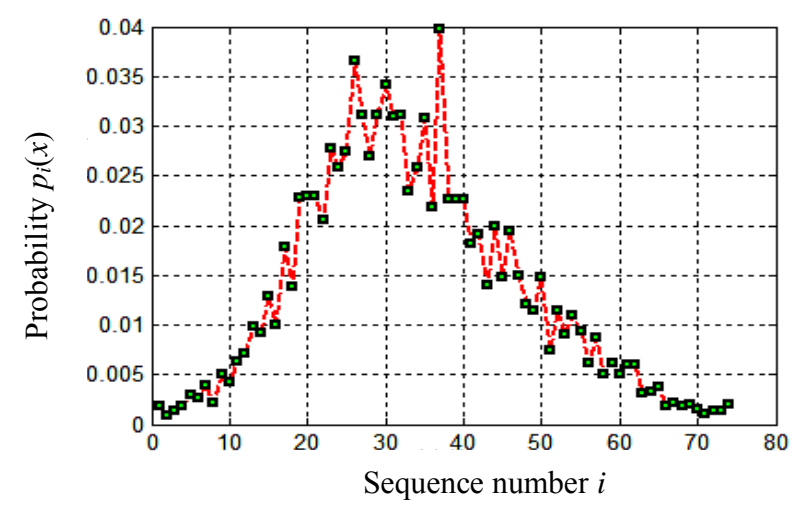

Fig. 6 Probability distribution of the friction torque (11r/min)

The mean of the friction torque of 5 sets of bearings in experiment is shown in Fig. 7. It concluded that under low speed, information entropy of the friction torque mounted up nonlinearly with increase of bearing rotating speed. Therefore, the variable uncertainty of rolling bearing friction torque can be characterized by information entropy.

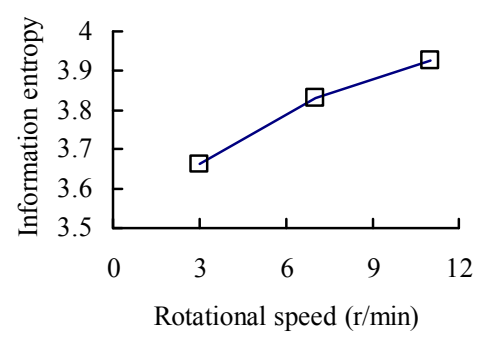

Fig. 7 The relationship of bearing rotational speed and information entropy 
Dynamic range of the mean of rolling bearing performances. Based on poor information theory, the Grey Bootstrap method is used to study the mean of dynamic fluctuant range (MDFR) of rolling bearing friction torque, and it reveals the variables uncertainty of bearing performances with the changing working condition. MDFR is defined as Eq. 2.

$$
U_{\text {mean }}=\frac{1}{T-m_{\mathrm{D}}} \sum_{k=m_{\mathrm{D}}+1}^{T}\left(X_{\mathrm{U}}(k)-X_{\mathrm{L}}(k)\right)
$$

where $T$ is the number of the data, $m_{D}$ is bootstrap sampling number, $X_{L}$ is the dynamic lower bound of the bearing performance which has been evaluated, $X_{U}$ is the upper bound of the bearing performance which has been evaluated.

The experimental conditions and experimental results are as well as the section 2.1. The MDFR of friction torque of 5 sets of test bearings is shown in Fig. 8. It can be seen that under low speed, the MDFR of friction torque presents a nonlinear upward trend with increase of the speed of bearings. So, the variable uncertainty of rolling bearing friction torque can be characterized by the MDFR.

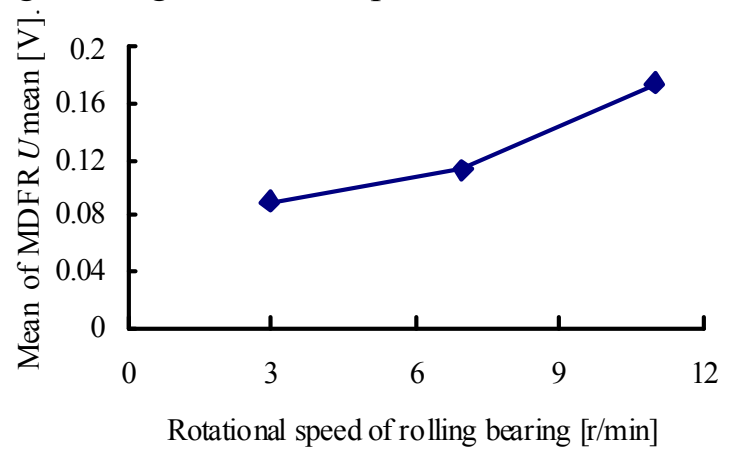

Fig. 8 The relationship of bearing rotational speed and MDFR

\section{The experimental evaluation of theoretical research on the structure of spatial relation of poor information}

Studies on variant gene of rolling bearing performances. In order to effectively real-time assess whether the rolling bearing performances are normal and the degree of failure of bearing performances during service, and take corresponding measures in time. Xia [10] proposed a method of evaluation for evolvement of a rolling bearing performance, using the fuzzy equivalence relation to represent the variant gene of nonlinearly evolutionary process of the rolling bearing performance as a time series based on phase space. For solving the problems of science and technology of the degree of rolling bearing failure in the field of real-time evaluation and prediction, this method integrates the chaotic time series theory and the fuzzy set theory, reconstructing the phase space for rolling bearing performance as a time series, meanwhile offers the rule of alarm to monitor online the variation process of bearing performance.

Via the transitive closure method of fuzzy set theory, the fuzzy equivalence relation between two phase trajectories $X_{g}(i)$ and $X_{g}(l)$ based on phase space can be obtained as Eq. 3.

$$
\mathbf{R}=\mathbf{S}^{q}=\left[r_{i l}(m, \tau)\right]_{M \times M}
$$

where $r_{i l}(m, \tau)$ is the fuzzy equivalence coefficient and $q$ is a positive integer. And there is the following Eq. 4.

$$
r_{i l}(m, \tau)=\left\{\begin{array}{l}
1 \quad ; i=l \\
r_{l i}(m, \tau) ; i \neq l
\end{array} \quad 0 \leq r_{i l}(m, \tau) \leq 1\right.
$$


Fuzzy equivalence coefficient $r_{i l}(m, \tau)$ and positive integer $q$ should meet the following relationship Eq. 5.

$$
\mathbf{S}^{q} \mathbf{O} \mathbf{S}^{q}=\mathbf{S}^{q} ; q=1,2, \mathrm{~L}
$$

where $C$ is the maximum and minimum operation $(\wedge, \vee)$.

The relation between $X_{g}(i)$ and $X_{g}(l)$ can be optimized if choosing the appropriate $m$ and $\tau$, therefore the optimum reconstruction phase space based on fuzzy equivalence relation is gained. At this point, the original dynamic characteristics of rolling bearing performances as a time series are recovered.

$$
u=\frac{1}{G} \sum_{g=1}^{G} u_{g}
$$

Eq. 6 is defined as the optimum measure of the fuzzy equivalence coefficient average based on phase space, hereinafter referred to as the optimum measure of fuzzy equivalence relation, which can eliminate the influence of random disturbance, and it can be seen that $0 \leq u \leq 1$. Where $u_{g}$ is the $g_{\text {th }}$ mean of the optimum fuzzy equivalence coefficient based on phase space, and $0 \leq u_{g} \leq 1$.

The optimum measure $u$ for fuzzy equivalence relation can be real and effective to characterize the variation process of rolling bearing performance, viz., the fuzzy equivalence relation $\mathrm{R}$ based on phase space can be seen as a variant gene that drives the evolution of rolling bearing performance.

Establishment of Information-Poor Relation Space. Based on the fuzzy set theory and the grey system theory, the poor information relation of bearing performance is investigated to explore the internal evolution mechanism and degree of variation of bearing performance. Through the discussion on the grey relation between time series $X_{i}$ and $X_{j}$, the optimum grey relation space can be evolved into the optimum fuzzy equivalence relation with experience confidence level, then the poor information space, viz., improved fuzzy equivalence relation space, is established. If describing the poor information relation in phase space, it is defined as the fuzzy equivalent relation based on phase space.

The relational space for poor information is defined as the following:

$$
\mathbf{R}=\left[\begin{array}{cc}
1 & r_{i j} \\
r_{j i} & 1
\end{array}\right]
$$

where,

$$
\begin{aligned}
& r_{i j}=r_{i j}\left(\eta, d_{i j \max }\right)=\left\{\begin{array}{l}
1-\frac{d_{i j \max }}{\eta} ; d_{i j \max } \in[0, \eta] \\
0 \quad ; d_{i j \max } \in[\eta, 1]
\end{array}\right. \\
& d_{i j \max }=\max _{\xi \rightarrow \xi^{*}} d_{i j}(\xi)
\end{aligned}
$$

where $d_{i j}$ is the difference of the membership for two time series, $\xi$ is the resolution coefficient, $\xi *$ is the optimal resolution coefficient, $\eta \in[0,1]$ is for the weight factor.

The empirical confidence level is defined as the following:

$$
P_{\mathrm{E}}=P_{\mathrm{E}}(\eta)=(1-\lambda \eta) \times 100 \%
$$

By means of information-poor relation space, the evolvement rule and the mutation mechanism of bearing performances can be researched. Poor information hypothesis testing is presented if introduction of the rejection region: if $r_{i j} \geq \lambda$, the properties of $X_{i}$ and $X_{j}$ are identical; and vise versa. Generally, the value of $\lambda$ is 0.5 . 


\section{Conclusions}

To predict the rolling bearing performance in time and ensure the rail transit safety is of great significance. The paper introduces information-poor system theory into test evaluation for rolling bearing performances, makes an in-depth study for the intrinsic evolvement mechanism of bearing performances.

The paper provides a new method to predict the evolvement of characteristics of rolling bearing performances. The information-poor relation space and variant gene of rolling bearing performances are established which lays a new theory basis for evaluating the evolvement of nonlinear dynamics characteristics of rolling bearing performances for the urban rail and traffic engineering. Poor information system is used to predict the rolling bearing performance, which realizes intelligent prediction and then ensures the safety of rail transit vehicles, fast operation.

\section{Acknowledgment}

This project is supported by National Natural Science Foundation of China (Grant Nos. 51475144 and 51075123).

\section{References}

[1] J. Ye, L.F. Yang, Development of bearing for rail transit vehicles, Bearing. 12 (2013) 61-65.

[2] Q. An, Y. Qin, and D.C. Chen, Research on fault diagnosis method for bearings of the urban rail transit vehicles, Chinese Railways. 11 (2014) 96-101.

[3] X.T. Xia, Forecasting method for product reliability along with performance data, Journal of Failure Analysis and Prevention. 12 (2012) 532-540.

[4] S.G. Shimizu, A new life theory for rolling bearings-by linkage between rolling contact fatigue and structural fatigue, Tribology Transactions. 55 (2012) 558-570.

[5] X.T. Xia, Z.Y. Wang, and H. Chang, Grey correlation of vibration with rolling bearing's machining quality, Journal of Aerospace Power. 20 (2005) 250-254.

[6] X.T. Xia, L. Chen, and F.N. Meng, Uncertainty of rolling bearing friction torque as data series using grey bootstrap method, Applied Mechanics and Materials. 44-47 (2011) 1125-1129.

[7] X.T. Xia, J. Fan, and L. Chen, Variation analysis of starting friction torque for slewing bearing based on poor information theory, Transactions of the Chinese Society of Agricultural Engineering. 28 (2012) 81-86.

[8] N. Oguma, Reliability design in rolling bearings, Journal of Japanese Society of Tribologists. 56 (2011) 673-679.

[9] F.Y. Cong, J. Chen, and Y.N. Pan, Kolmogorov-Smirnov test for rolling bearing performance degradation assessment and prognosis, Journal of Vibration and Control. 17 (2011) 1337-1347.

[10] X.T. Xia, L. Chen, Fuzzy chaos method for evaluation of nonlinearly evolutionary process of rolling bearing performance, Measurement. 46 (2013) 1349-1354. 